This item was submitted to Loughborough's Research Repository by the author.

Items in Figshare are protected by copyright, with all rights reserved, unless otherwise indicated.

\title{
Peak-to-average power ratio mitigation in quasi-orthogonal space time block coded MIMO-OFDM systems using selective mapping.
}

PLEASE CITE THE PUBLISHED VERSION

\section{PUBLISHER}

(C) IEEE

\section{VERSION}

VoR (Version of Record)

\section{LICENCE}

CC BY-NC-ND 4.0

\section{REPOSITORY RECORD}

Alharbi, Faisal S., and Jonathon Chambers. 2019. "Peak-to-average Power Ratio Mitigation in Quasiorthogonal Space Time Block Coded MIMO-OFDM Systems Using Selective Mapping.". figshare. https://hdl.handle.net/2134/5590. 
This item was submitted to Loughborough's Institutional Repository (https://dspace.lboro.ac.uk/) by the author and is made available under the following Creative Commons Licence conditions.

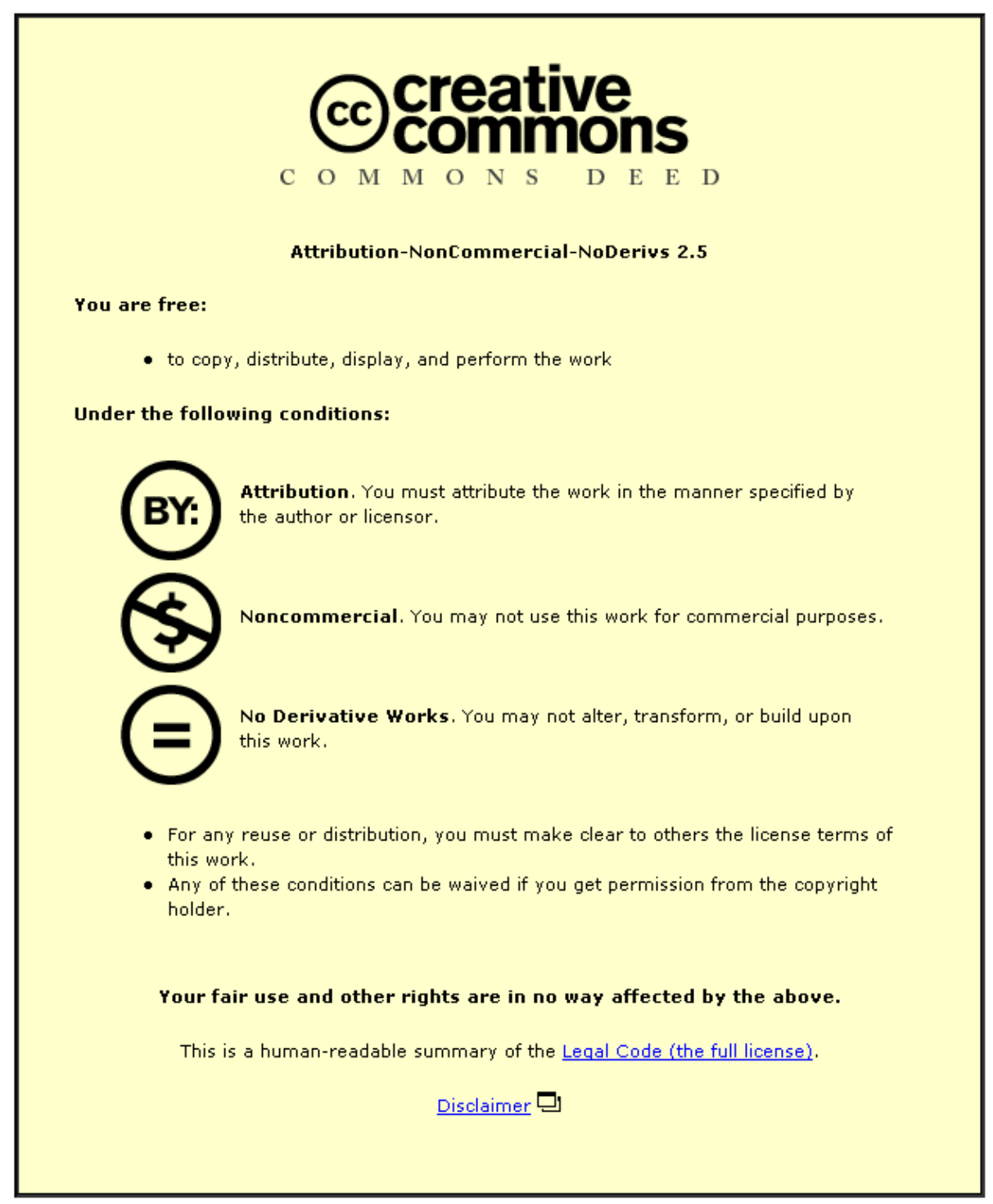

For the full text of this licence, please go to: http://creativecommons.org/licenses/by-nc-nd/2.5/ 


\title{
PEAK-TO-AVERAGE POWER RATIO MITIGATION IN QUASI-ORTHOGONAL SPACE TIME BLOCK CODED MIMO-OFDM SYSTEMS USING SELECTIVE MAPPING
}

\author{
F.S. Alharbi and J.A.Chambers \\ Advanced Signal Processing Group Dept of Electronic and Electrical Engineering \\ Loughborough University, LE11 3TU, England. \\ Email: f.alharbi@lboro.ac.uk, j.a.chambers@lboro.ac.uk
}

\begin{abstract}
A study of a peak-to-average power ratio (PAPR) reduction scheme for quasi-orthogonal spacetime block coded multi-input multi-output (MIMO) orthogonal frequency division multiplexing (OFDM) systems based on selective mapping (SLM) is presented. The reduction technique is based upon combining the PAPRs of the transmission blocks from four antennas and exploits the associated antenna diversity gain to mitigate errors in the transmission of the side information (SI) necessary for SLM. Simulation studies are presented which show the cumulative complementary distribution functions (CCDFs) with and without the combining scheme and bit error rates of the overall system. Comparisons are made with single antenna and conventional OFDM schemes.
\end{abstract}

Index Terms- peak-to-average power ratio (PAPR), orthogonal frequency-division multiplexing (OFDM), selective mapping (SLM), cumulative complementary distribution functions (CCDFs), side information (SI).

\section{INTRODUCTION}

Orthogonal frequency division multiplexing (OFDM) is an efficient digital modulation technique for high data rate transmission especially in slowly fading and multipath channels. This technique has a relatively simple implementation based on the fast discrete Fourier transform, which avoids complicated equalization algorithms. OFDM divides the available spectrum into many subcarriers, instead of using only one carrier, and each one is modulated by a low rate data stream [8]. Despite the widespread acceptance of OFDM in systems such as WiFi/WiMax [7], it has an inherent drawback of high peak-to-average power ratio (PAPR) which can increase the complexity of the digital-to-analog converter in the transmission and increase the cost of the power amplifier. Given these facts, several researchers have extensively examined PAPR reduction in single-input single-output (SISO) systems (see, for instance, [1]- [3]). In the selected mapping approach [2] the frame to be transmitted is multiplied by a number of preselected phase signals such that the resulting PAPR is modified and then that mapping which yields the smallest PAPR is the one which is chosen; and therefore the receiver must know which out of the candidate phase waveforms is the one which was actually used. For high data rate wireless wideband applications, multiple-input multiple-output (MIMO) transmission combined with OFDM is being considered in a large number of current technology applications. If the PAPR reduction schemes in SISO are directly applied to MIMO, the complexity and redundancy increases proportional to the number of transmit antennas, which is not desired. In a traditional single antenna system, multi-path propagation can be a problem as it causes inter-symbol interference (ISI). As explained above the transmission of side information (SI) is critical in the selective mapping scheme, which when corrupted leads to a high risk of interpreting all of the transmitted symbols incorrectly. It is thus advisable to protect such information using the diversity gain afforded by MIMO transmission. By employing a MIMO system, multiple spatial channels are created and it is unlikely that all the channels will fade simultaneously. Recent work in [4] is based upon exploiting these advantages. However, this work is limited to two transmit and one receive antenna.

In this paper for MIMO-OFDM, we extend the proposed technique in [4] and study the PAPR performance of a quasi-orthogonal space-time block code (QO-STBC) using a joint SLM technique for PAPR reduction. Instead of applying individual SLM to each antenna, the sequence with the lowest PAPR over four transmit antennas is selected. Using the MIMO-SLM can improve the overall bit-error rate (BER) performance and increase the reliability of SI at the expense of the PAPR reduction gain. The interaction between the feedback necessary for maximum diversity gain in the full rate QO-STBC scheme we exploit and the proposed SLM scheme is also discussed. In the next section, we introduce the SLM for a single antenna situation. In Section III, the notion of block quasi-orthogonal space-time coding is presented. Simulation results are provided in Section IV. Finally, Section $\mathrm{V}$ gives concluding remarks.

\section{SINGLE ANTENNA SLM IN MIMO-OFDM SYSTEMS}

The basic principle of the SLM technique is that if there are M different ways of representing the same underlying signal information, $\mathbf{C}_{m}, m=1,2, \ldots, M$, then, only one of these is likely to have the lowest PAPR 
when converted by the inverse Fourier transform (IFFT). The heart of the scheme is the methods to derive the different ways to represent the original source signal.

The $M$ subvectors within $\mathbf{C}$ are weighted elementwise by the components of the generally complex vectors $b_{i}, i=1, \ldots, M$ to form $\mathbf{C}_{i}, i=1, \ldots, M$. These are then applied to the IFFT and the PAPR of their outputs are calculated and the final sequence is chosen to be the one with the lowest PAPR. The elements of the $b_{i}$ sequences are of the form of complex phases $e^{j \phi}$, typically $\phi \in( \pm 1, \pm j)$ for computational simplicity, since phase shifts by multiplies of $\pi / 2$ can be implemented without any multiplications. To recover the data, the receiver must know which sequence has been used; this can be transmitted as SI. For the case where the number of carriers is sufficiently large, the time domain signal sampled at Nyquist rate is approximately zeromean complex Gaussian based on adopting the central limit theorem. Therefore, the amplitude of these samples has a Rayleigh distribution and the probability that the PAPR exceeds a given threshold $\gamma$ can be expressed as:

$$
\operatorname{Pr}(P A P R>\gamma)=1-\left(1-e^{-\gamma}\right)^{N}
$$

where $\mathrm{N}$ is the number of subcarriers within the OFDM frame. Then, the probability that this happens on $M$ independent sequences is:

$$
\operatorname{Pr}\left(P A P R_{\text {low }}>\gamma\right)=\operatorname{Pr}(P A P R>\gamma)^{M}
$$

where $P A P R_{\text {low }}$ is the frame with the lowest PAPR. The underlying idea of the combined scheme is to exploit this result and to form an overall vector for the MIMO system which is composed of the sub-blocks for each antenna, so the overall PAPR can be calculated in (2). In the work in [4], it was restricted to two antennas and Alamouti's space-time block coding scheme. In this work, we extend it to QO-STBC for four antennas due to increased diversity at full rate which is possible with closed loop operation.

\section{QO-STBCS SLM IN MIMO-OFDM SYSTEMS}

In this work we are considering four antenna systems and the block code matrix is defined by [5]:

$$
\mathbf{G}(\mathbf{S})=\left(\begin{array}{cccc}
\mathbf{S}_{1} & \mathbf{S}_{2} & \mathbf{S}_{3} & \mathbf{S}_{4} \\
-\mathbf{S}_{2}^{*} & \mathbf{S}_{1}^{*} & -\mathbf{S}_{4}^{*} & \mathbf{S}_{3}^{*} \\
-\mathbf{S}_{3}^{*} & -\mathbf{S}_{4}^{*} & \mathbf{S}_{1}^{*} & \mathbf{S}_{2}^{*} \\
\mathbf{S}_{4} & -\mathbf{S}_{3} & -\mathbf{S}_{2} & \mathbf{S}_{1}
\end{array}\right)
$$

where the operation $(.)^{*}$ denotes complex conjugation. The transmit matrix $\mathbf{G}(\mathbf{S})$ corresponds to four transmit antennas transmitting a symbol block of four OFDM symbol intervals. The $n^{\text {th }}$ column of this matrix corresponds to the blocks transmitted from the $i^{t h}$ antenna at consecutive symbol intervals. Feedback is also assumed as in [5] to obtain full diversity and rate performance. The time-domain OFDM signal, without cyclic prefix, transmitted from the $i^{t h}$ transmit antenna is represented algebraically as:

$$
s_{i}(t)=\sum_{n=0}^{N-1} c_{i, n} e^{j 2 \pi t n / N}, 0 \leq t \leq N-1
$$

where $s_{i}, i=1,2,3,4$ denote the complex valued time-domain transmitted symbols and $c_{i, n}=\left[\mathbf{S}_{i}\right]_{n}$ is the complex symbol of transmitter $i$ at the $n^{\text {th }}$ subcarrier in the frequency domain. We assume that the above matrix block code is transmitted in the form of quaternary phase-shift keying (QPSK) symbols through a frequency selective channel and the signal is received with only one receive antenna. Between each transmitter and the one receive antenna, we are assuming a multipath channel and the coefficients of that channel are modelled by independent complex valued random variables with zero mean and unity variance. We also assume that each multipath channel is quasi-static, i.e. it remains constant over the block interval of the quasi-orthogonal transmission. The PAPR associated with a MIMO-OFDM system can be defined as [6]:

$$
P A P R=\frac{\max _{i, t}\left|s_{i}(t)\right|^{2}}{E_{i, t}\left[\left|s_{i}(t)\right|^{2}\right]}
$$

where $E_{i, t}[$.$] denotes the expectation over transmit antennas and time. It is natural to individually apply SLM$ in (Section 1) to each antenna in QO-STBC. The OFDM symbol is mapped to a number of $M$ independent candidate symbols representing the same information. Then, the candidates $\mathbf{C}_{i}=\left[c_{0}, \ldots, c_{N-1}\right]$ are generated by multiplying carrier-wise the original OFDM frame with $M$ phase vectors $b_{M}=\left[b_{0}, \ldots, b_{N-1}\right],\left[b_{M}\right]_{v}=e^{j \varphi_{v}}$, and $\varphi_{v} \in( \pm 1, \pm j)$ is randomly selected, the chosen phases are assumed to be known to both transmitter and receiver. In order to recover the data at the receiver SI bits, of length $S=\left[\log _{2}(M)\right]$, have to be transmitted to indicate which sequence out of the $b_{M} s$ was used in the transmitter. The detection probability of this SI can be given by: 


$$
P_{d}=1-\left[\log _{2} M\right] \times \zeta
$$

where

$$
\zeta=\frac{(1-\beta)}{2} \sum_{d=0}^{D-1}\left(\begin{array}{c}
D-1+d \\
d
\end{array}\right)\left(\frac{1+\beta}{2}\right)^{d} \text { and } \beta=\frac{1}{\sqrt{1+1 / S N R}}
$$

where D represents the number of diversity channels carrying the same SI [6]. In the proposed combined scheme, SI is best transmitted over evenly distributed locations in frequency. In this work we use QPSK modulation which means each carries $\log _{2} Q$ SI bits and the OFDM frame is divided into four uniformly separated regions across the frequency range as follows:

$$
n_{i}= \begin{cases}(i-1) \frac{N}{L_{t}} & \text { if } i \leq \frac{L_{t}}{4} \\ N-\left[\frac{\frac{N}{2} S}{\log _{2} Q}\right]-\left(\frac{L_{t}}{2}-i\right) & \text { if } \frac{L_{t}}{4} \leq i<\frac{L_{t}}{4}+1 \\ N-\left[\frac{\frac{N}{2} S}{\log _{2} Q}\right]-\left(\frac{L_{t}}{2}+1-i\right) & \text { if } \frac{L_{t}}{4}+1 \leq i<L_{t} \\ N+\left[\frac{S}{\log _{2} Q}\right]-\left(\frac{3 L_{t}}{2}-i\right) & \text { if } i \geq L_{t}\end{cases}
$$

where $L_{t}$ denotes the number of the transmit antennas. By doing this, we are positioning the SI as far apart across frequency as possible so that we maximize the frequency diversity gain and that same SI is transmitted on all of the antennas which maximizes the spatial diversity. It should be noted that the PAPR of a continuous time OFDM signal cannot generally be computed precisely by using the Nyquist sampling rate. In this case, signal peaks can be missed and PAPR reduction estimates are unduly optimistic. Using a longer IFFT, i.e. interpolation, typically four times oversampling is effective in ensuring that PAPR is accurately estimated. In the combined SLM approach, the selection among those $M$ random sequences is made according to the average PAPR across the antennas and the corresponding cumulative complementary distribution function (CCDF) of the best sequence is:

$$
\operatorname{Pr}\left(P A P R_{\text {low }}>\gamma\right)=\left[1-\left(1-e^{-\gamma}\right)^{N \times L_{t}}\right]^{M}
$$

where $\gamma$ is the threshold of PAPR. The mathematical relationship between (6) and (9) could be studied further. Next, we consider a numerical evaluation of the performance of this approach.

\section{Simulation Results}

We initially consider the CCDF performance of our scheme and compare the analytical results with numerical results. In particular, Fig. 1 confirms that as the possible number of subsequences $M$ changes from 1 to 4 , with an oversampling factor of 1 ; the curve slope is increased. Also, it can be seen that the analytical curves according to (9) are identical to the simulation results. In this figure, consider when the probability of $P A P R>\gamma=0.001$ in this case when $M=1$ the value of $\gamma>10 \mathrm{~dB}$. However, when $M=4$ the value of $\gamma$ is between 8 and $9 \mathrm{~dB}$ demonstrating at least $1 \mathrm{~dB}$ reduction.

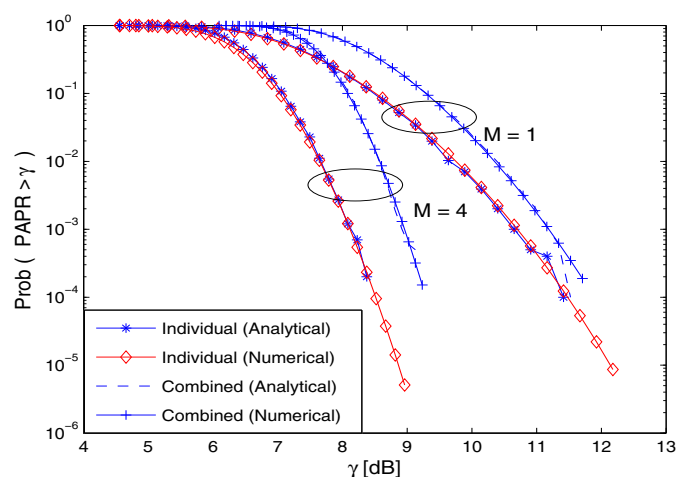

Fig. 1. CCDF of PAPR, the frame with lowest average PAPR is selected out of $M$ statistically independent frames for both single and combined SLM methods, $L_{t}=4$ transmit antennas, $N=128$, and QPSK modulates each carrier, oversampling factor of unity.

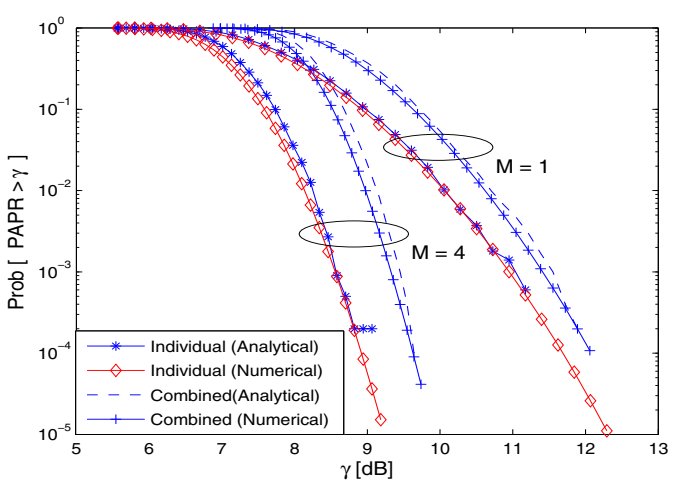

Fig. 2. As Fig. 1. except for an oversampling factor of

Fig. 2 generates a more accurate representation of the true PAPR through oversampling by a factor of 4 . Importantly, in case of QO-STBCs for example, the minimum PAPR threshold that was found is different. In 
the case of no interpolation for the combined scheme, the minimum PAPR achieved was 9dB for the combined QO-STBCs. However, with interpolation the new minimum PAPR changed to $9.5 \mathrm{~dB}$ for the same number of antennas. This $0.5 \mathrm{~dB}$ difference corresponds to properly finding the peaks in the time-domain signal through the oversampling operation. In Fig. 3 we investigate the BER performance of the complete conventional baseband OFDM system. We compare this with our proposed combined closed-loop phase scheme and open-loop scheme both for four transmit and one receive antennas over frequency selective channels, $N=128$ and $M=2$. It is noted that, at BER $=0.001$ the closed-loop scheme needs $E_{b} / N_{o}$ of approximately $11 \mathrm{~dB}$ while this value increased to at least $17 \mathrm{~dB}$ with the conventional scheme. Moreover, the overall BER performance matches the perfect SI detection when exploiting four antenna diversity.

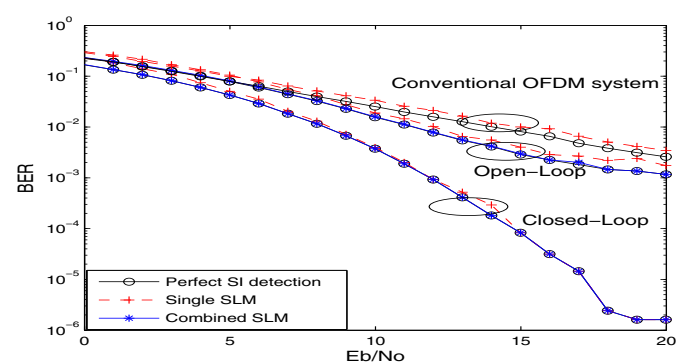

Fig. 3. BER comparison with respect to side information for both single and combined SLM methods of QO-STBCs with and without feedback, $N=128$ and $M=2$.

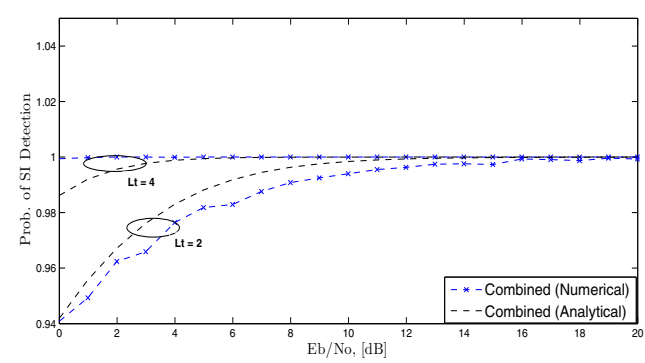

Fig. 4. Probability of side information detection using SLM method for $2 \times 1$ STBC-SLM and a combined $4 \times 1$ QO-STBC, $N=128$ and $M=2$.

For the final simulation, in Fig. 4 we provide the probability of detection of SI for both $L_{t}=2$ MIMOSTBC and $L_{t}=4$ QO-STBCs based on the SLM technique. We observe that when $L_{t}=2$, the numerical and the analytical results give a probability of SI detection around 0.95 at $E_{b} / N_{o}=2 \mathrm{~dB}$ and with $L_{t}=4$, this probability is increased by approximately $4 \%$. It is evident that this scheme is a promising solution for considerably increasing the probability of SI detection. The QO-STBC scheme requires to operate in closed-loop mode in order to have full diversity and full rate. To achieve this, feedback is required from the receiver to the transmitter. When such a feedback is used at the transmitter, the transmitted symbols are rotated by a phase value. This operation should not be performed in isolation from the phase rotations that we are applying in terms of the PAPR mitigation scheme. Therefore, future work will be looking at the combination of the feedback together with the PAPR mitigation.

\section{Conclusion}

In this paper, a combined SLM approach was proposed for PAPR mitigation in MIMO-OFDM transmission over four transmit antenna systems which exploits closed loop operation with a full rate and full diversity QOSTBC. Performance improvement was demonstrated in terms of comparison with a conventional OFDM SLM scheme in terms of average BER and probability of SI detection.

\section{REFERENCES}

[1] A.D.S. Jayalath and C. Tellambura, "Adaptive PTS approach for reduction of peak-to-average power ratio of OFDM signal,” Electronic Lett., vol.36, no.14, pp.1226-1228, July 2000.

[2] R.W. Bauml, R.F.H. Fischer, and J.B. Huber, "Reducing the peak-to-average power ratio of multicarrier modulation by selected mapping," Electronic Lett., vol.32, no.22, pp.2056-2057, October 1996.

[3] Li. Xiaodong and L.J.Jr. Cimini, "Effect of Clipping and Filtering on the Performance of OFDM," Electronic Trans., vol.2, no.5, pp.131-133, May 1997.

[4] Y.-L. Lee, Y.-H. You, W.-G. Joen, J.-H. Paik, and H.-K. Song, "Peak-to-average power ratio in MIMOOFDM systems using selective mapping," IEEE Commun. Lett., vol.7, no.12, pp.575-577, December 2003.

[5] C.Toker, S.Lambotharan, and J.A. Chambers, "Closed-Loop Quasi-Orthogonal STBCs and Their Performance in Multipath Fading Enviroments and When Combined With Turbo Codes," IEEE Trans. Commun., vol.3, no.6, pp.1890-1896, November 2004.

[6] J.G. Proakis, Digital Communications. New York: McGraw-Hill, 1989.

[7] H. Yagoobi, "Scalable OFDMA Physical Layer in IEEE 802.16 WirelessMan," Intel Technology Journal, vol.8, pp. 201-212, August 2004.

[8] A. R. Bahai, and B. R. Saltzaberg, Multi-Carrier Digital Comunications:Theory and Applications of OFDM. New York: Kluwer Academic/Plenum Publishers, 1999. 